\title{
The Venus Emissivity Mapper (VEM) concept
}

Joern Helbert, Dennis Wendler, Ingo Walter, Thomas Widemann, Emmanuel Marcq, et al.

Joern Helbert, Dennis Wendler, Ingo Walter, Thomas Widemann,

Emmanuel Marcq, Gabriel Guignan, Sabrina Ferrari, Alessandro Maturilli, Nils Mueller, David Kappel, Judit Jaenchen, Mario D'Amore, Anko Boerner, Darby Dyar, Gabriele E. Arnold, Suzanne Smrekar, "The Venus Emissivity Mapper (VEM) concept," Proc. SPIE 9973, Infrared Remote Sensing and Instrumentation XXIV, 99730R (14 September 2016); doi:

$10.1117 / 12.2237568$

EDIE Event: SPIE Optical Engineering + Applications, 2016, San Diego, California, United States 


\title{
The Venus Emissivity Mapper Concept
}

Joern Helbert ${ }^{*}$, Dennis Wendler ${ }^{\mathrm{a}}$, Ingo Walter ${ }^{\mathrm{a}}$, Thomas Widemann ${ }^{\mathrm{B}}$, Emmanuel Marcq ${ }^{\mathrm{c}}$, Gabriel Guignan $^{c}$, Sabrina Ferrari ${ }^{\mathrm{d}}$, Alessandro Maturillia, Nils Muellere, David Kappela , Judit Jaenchen ${ }^{\mathrm{a}}$, Mario D’Amore ${ }^{\mathrm{a}}$, Anko Boerner ${ }^{\mathrm{a}}$, Darby Dyar ${ }^{\mathrm{f}}$, Gabriele E. Arnold ${ }^{\mathrm{a}}$, Suzanne Smrekar ${ }^{\mathrm{e}}$

${ }^{a}$ Deutsches Zentrum für Luft- und Raumfahrt e.V. (Germany); 'bab. d'Etudes Spatiales et d'Instrumentation en Astrophysique (France); ${ }^{c}$ LATMOS (France); ${ }^{\mathrm{d} U n i v}$. degli Studi di Pavia (Italy); ${ }^{\mathrm{e}}$ California Institute of Technology (USA) and Jet Propulsion Lab. (USA); ${ }^{\mathrm{f}}$ Mount Holyoke College (USA)

\begin{abstract}
Based on experience gained from using the VIRTIS instrument on Venus Express to observe the surface of Venus and the new high temperature laboratory experiments, we have developed the multispectral Venus Emissivity Mapper (VEM) to study the surface of Venus. VEM imposes minimal requirements on the spacecraft and mission design and can therefore be added to any future Venus mission. Ideally, the VEM instrument will be combined with a high-resolution radar mapper to provide accurate topographic information, as it will be the case for the NASA Discovery VERITAS mission or the ESA EnVision M5 proposal.
\end{abstract}

Keywords: Venus, near infrared, spectroscopy

\section{INTRODUCTION}

The permanent cloud cover of Venus prohibits observations of the surface with traditional imaging techniques over the entire visible spectral range. Fortunately, Venus' atmospheric gases are largely transparent in narrow spectral windows near $1 \mu \mathrm{m}$. Ground observers were the first to successfully use these windows, followed by spacecraft observations during the flyby of the Galileo mission on its way to Jupiter and most recently from Venus orbit by ESA's Venus Express with the VMC and VIRTIS instruments. Analyses of VIRTIS measurements have successfully demonstrated that surface information can be extracted from these windows, but the design of the instrument limited its use for more in-depth surface investigations.

Based on experience gained from using VIRTIS to observe the surface of Venus and new high temperature laboratory experiments currently performed at the Planetary Spectroscopy Laboratory of DLR, we have designed the multi-spectral Venus Emissivity Mapper (VEM). Observations from VIRTIS have revealed surface emissivity variations correlated with geological features, but existing data sets contain only three spectral channels. VEM is optimized to map the surface composition and texture, and to search for active volcanism using the narrow atmospheric windows, building on lessons from prior instrumentation and methodology. It offers an opportunity to gain important information about surface mineralogy and texture by virtue of having six different channels for surface mapping.

VEM is focused mainly on observing the surface, mapping in all near-IR atmospheric windows using filters with spectral characteristics optimized for the wavelengths and widths of those windows. It also observes bands necessary for correcting atmospheric effects; these bands also provide valuable scientific data on composition as well as altitude and size distribution of the cloud particles, and on $\mathrm{H} 2 \mathrm{O}$ vapor abundance variations in the lowest $15 \mathrm{~km}$ of the atmosphere.

In combination with a high-resolution radar mapper that provides accurate topographic data as planned for the NASA VERITAS mission [1] or for the ESA EnVision mission [2], VEM will provide new insights into current properties and the geologic evolution of Venus.

*joern.helbert@dlr.de; phone +49 30 67055-319; http://www.dlr.de/pf/desktopdefault.aspx/tabid-10866/19013_read-44266/

Infrared Remote Sensing and Instrumentation XXIV, edited by Marija Strojnik, Proc. of SPIE Vol. 9973, 99730R - (c) 2016 SPIE · CCC code: 0277-786X/16/\$18 · doi: 10.1117/12.2237568 


\section{THE VEM CONCEPT}

\subsection{System design}

VEM is focused mainly on observing the surface, mapping in all near-IR atmospheric windows using filters with spectral characteristics optimized for the wavelengths and widths of those windows. It also observes bands necessary for correcting atmospheric effects [4-7]; these bands also provide valuable scientific data on cloud thickness, cloud opacity variations, article size distribution and $\mathrm{H}_{2} \mathrm{O}$ abundance variations in the lowest $15 \mathrm{~km}$ of the atmosphere [6-7].

VEM is a pushbroom multispectral imaging system. A baffle protects VEM from scattered light. Telecentric optics image the scene onto the filter array. This image is relayed by a three-lenses objective onto the detector. VEM is a monoblock configuration. VEM's optical sub-system (VEMO) sits on top of the electronics compartment, which includes the Instrument Controller (VEMIC) and the power supply (VEMPS). A two-stage baffle (VEMBA) protects VEM from scattered light.

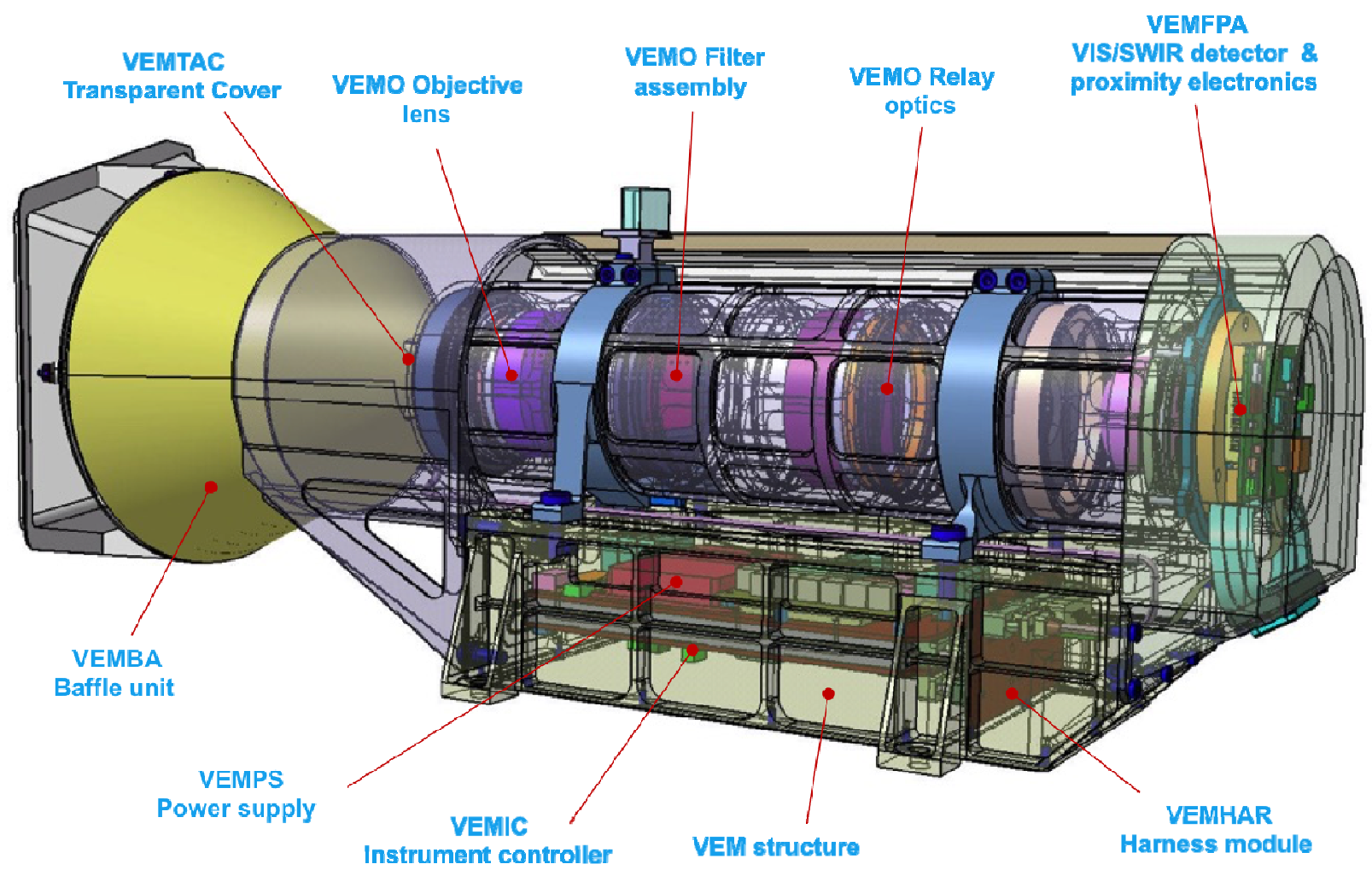

Figure 1 VEM design with strong design heritage from the MERTIS instrument on BepiColombo

VEMO is a telecentric optics consisting of only three lenses. A single lens objective relays this image onto the filter array (Figure 2). A $45^{\circ} \mathrm{FOV}$ yields a swath width of $207 \mathrm{~km}$ at an altitude of $250 \mathrm{~km}$, providing a thorough sampling of surface emissivity and orbit-orbit repeat coverage. VEM oversamples in science phase II at $10 \mathrm{~km}$ per pixel to account for local atmospheric water vapor variations and cloud removal. 


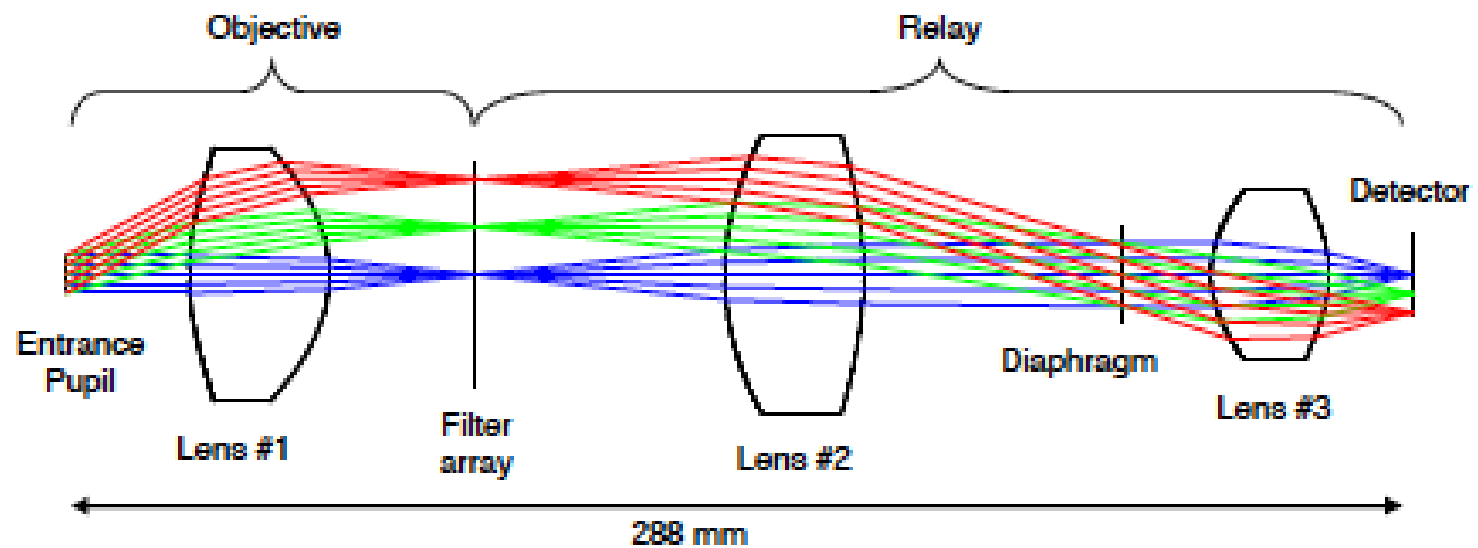

Figure 2 VEM optics (VEMO) uses only three lenses and a filter array as dispersive element

VEM uses a multilayered dielectric-coating ultra-narrow-band filter array to split the light into 14 bands. The filter array is located at an intermediary focus of the optical path. Each band is imaged by a two lenses relay optic onto 33x640-pixel rows on the FPA. Surface mineralogy bands are spatially interleaved between cloud bands to provide before and after calibration.

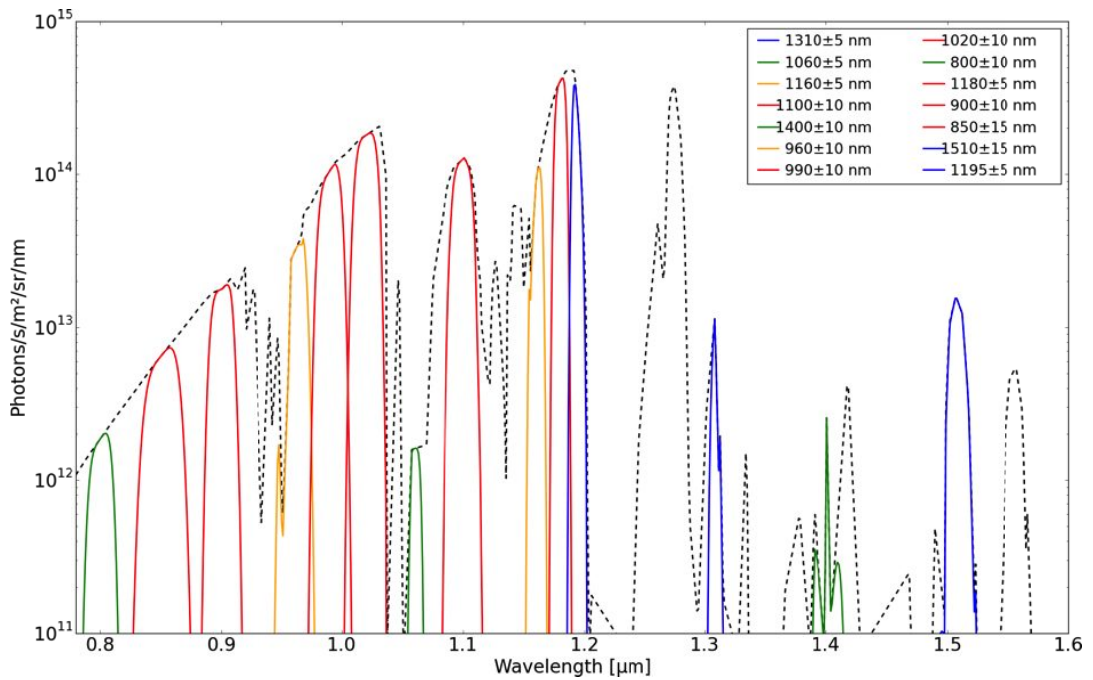

Figure 3 VEM current filter selection, the dashed envelope is a synthetic Venus night side spectrum [3] - Red are surface bands, blue bands for cloud correction, green stray light correction and yellow bands to detect water vapor

Core element of the focal plane array VEMFPA) is the 640x512 pixel Xenics XSW-640 InGaAs detector. The FOV is $30^{\circ} \times 45^{\circ}$; each $20-\mu \mathrm{m}$-pitch pixel sees a $0.07^{\circ} \times 0.07^{\circ} \mathrm{FOV}$. An integrated thermoelectric cooler is used to stabilize the working point of the detector. The FPA requires no cryogenic cooling avoiding a single point failure. The frontend electronics uses the highly integrated AFE device LM98640QML-SP, a full qualified (radiation tolerant), 14 bit, 5 MSPS to 40 MSPS, dual channel, complete Analog Front End. It was especially designed for digital imaging applications by Texas instruments. 


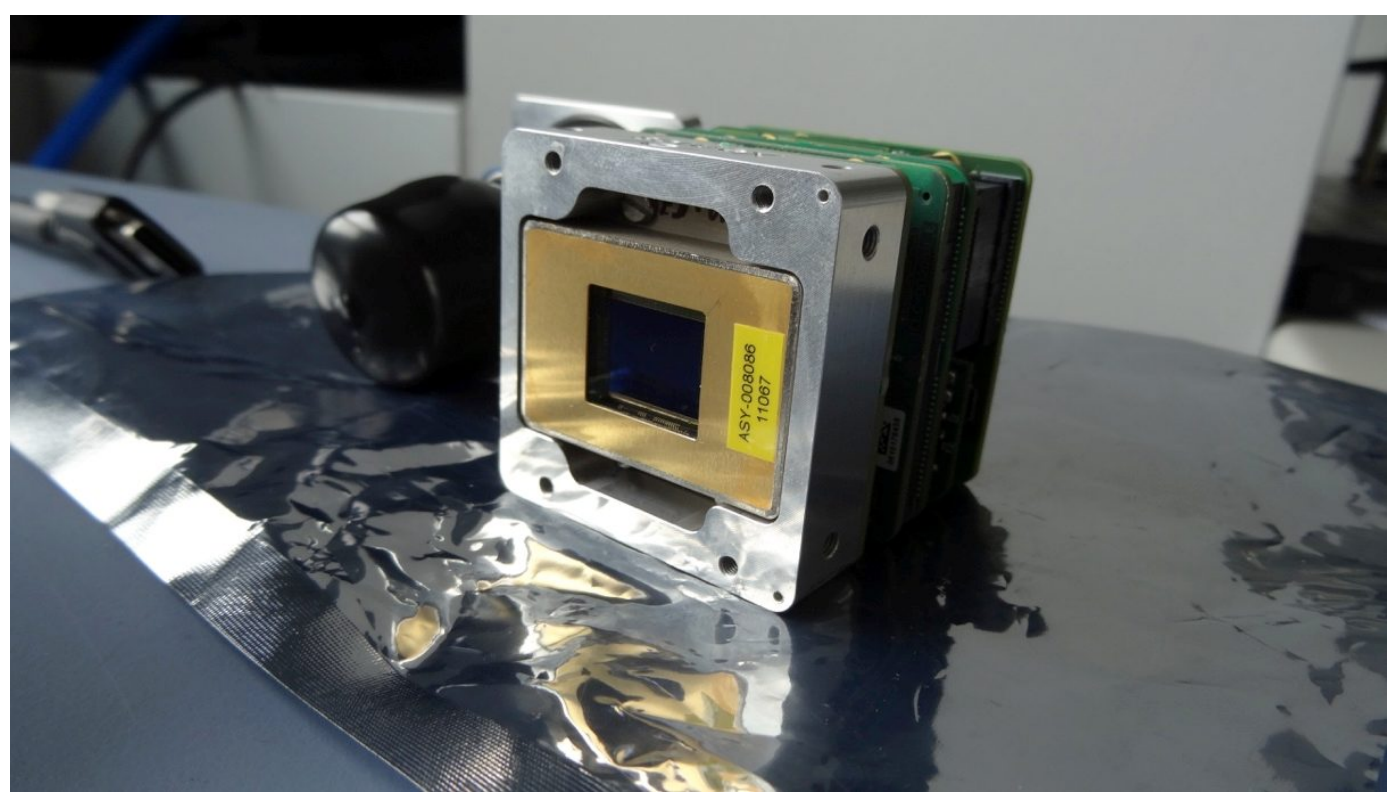

Figure 4 Commercial version of the Xenics XSW-640 InGaAs detector

The ICU (VEMIC) provides the electrical interface to the spacecraft and internal units, processes the detector data, performs binning, TDI and compression, and controls the sub-systems including digital and analog housekeeping electronics for acquiring VEM scientific and operational HKs. The ICU consists of one ACTEL FPGA including LEON-FT processor core, 128 Mbyte SDRAM and 2 MByte EEPROM. The design of the Power Supply Unit (VEMPS) is determined by the power requirements of VEMFPA and VEMIC. Baseline for VEMPS is to use the heritage of electrical and mechanical design of the proven MERTIS PSU. This PSU consists mainly of using a DC/DC converter of Interpoint's SMRT family expanded by external LC-filters and a special circuit for supporting the TEC on the VEMFPA.

VEMBA is a two-stage baffle with a light-weight front part which is mostly screening the $\mathrm{S} / \mathrm{C}$ but might get sun (Aluminum, white ceramic coating, high temperature range) and a back part suppressing straylight (3D selective laser melting of $\mathrm{TiAl}_{6} \mathrm{~V}_{4}$ - multiple black vanes, outside gold).

An optional transparent aperture cover (VEMTAC) using fused silica can protect the optics in cruise. Observations can be performed through the window. In the case of contamination on the window it is opened used a spring-loaded one-shot mechanism.

\subsection{VEM development approach}

VEM is following the successful MERTIS development approach [4]. Following an early breadboard [5] risks are mitigated and eliminated early with a lab prototype in Phase A that will be extended to a engineering prototype in Phase B. Following the risk mitigation a full qualification model is built undergoing all relevant test at qualification level. 


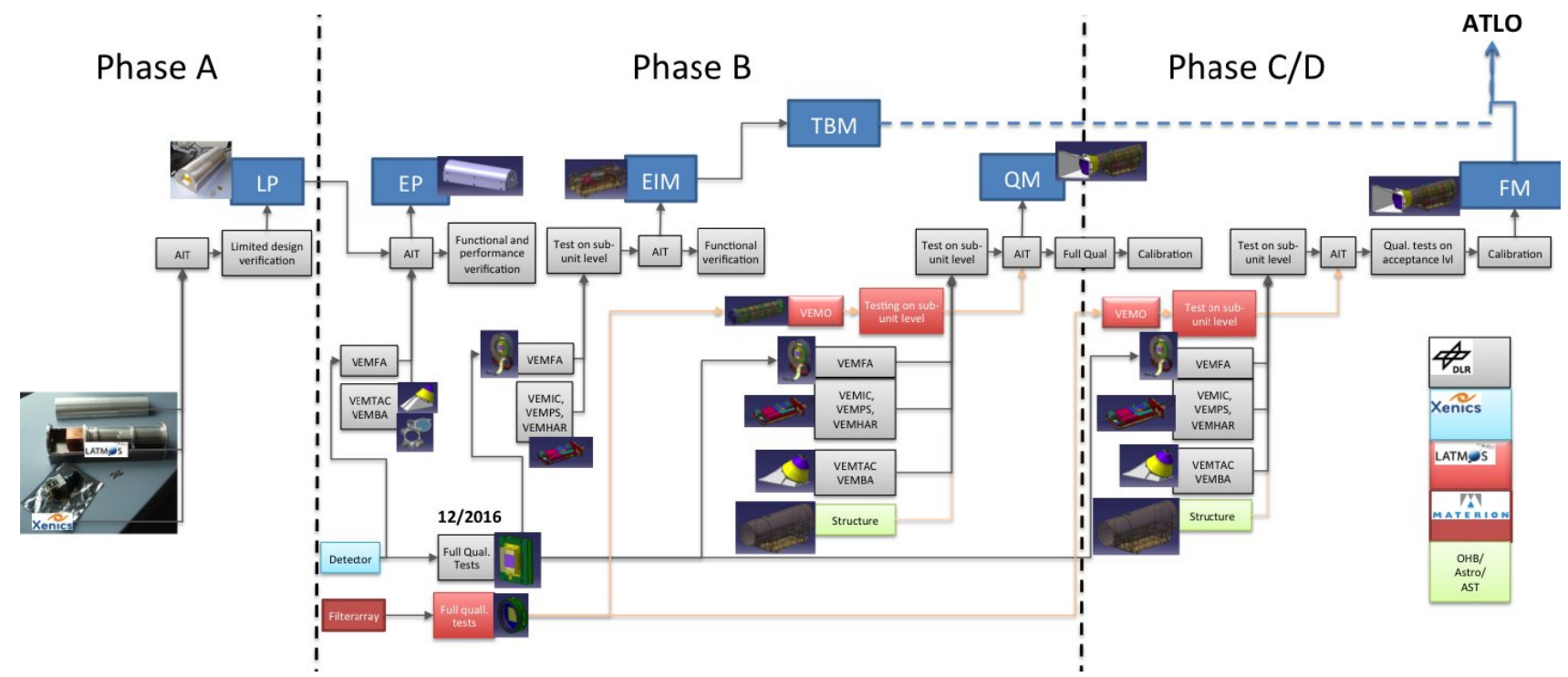

Figure 5 Sample AIT flow for the VEM instrument (LP - Laboratory Prototype, EP - Engineering Prototype, EIM - Electrical Interface Model, TBM - Testbench Model, QM - Qualification Model, FM - Flight Model)

\subsection{VEM laboratory prototype}

During Phase A for the NASA Discovery proposal VERITAS [1] a laboratory prototype (LP) of the VEM instrument has been integrated (Figure 5). This prototype includes a development version of the VEM optics using radiation hard glass and a simplified filter array. The detector used is the commercial version of the flight detector (Figure 3 ) and off the shelf front-end electronics is used with a laptop as electrical ground support equipment (EGSE). Form and function are already representative of the top part of the VEM flight instrument. The LP has been integrated and passed functional verification in July 2016. Currently performance testing is ongoing at the Planetary Spectroscopy Laboratory (see section 3) of DLR

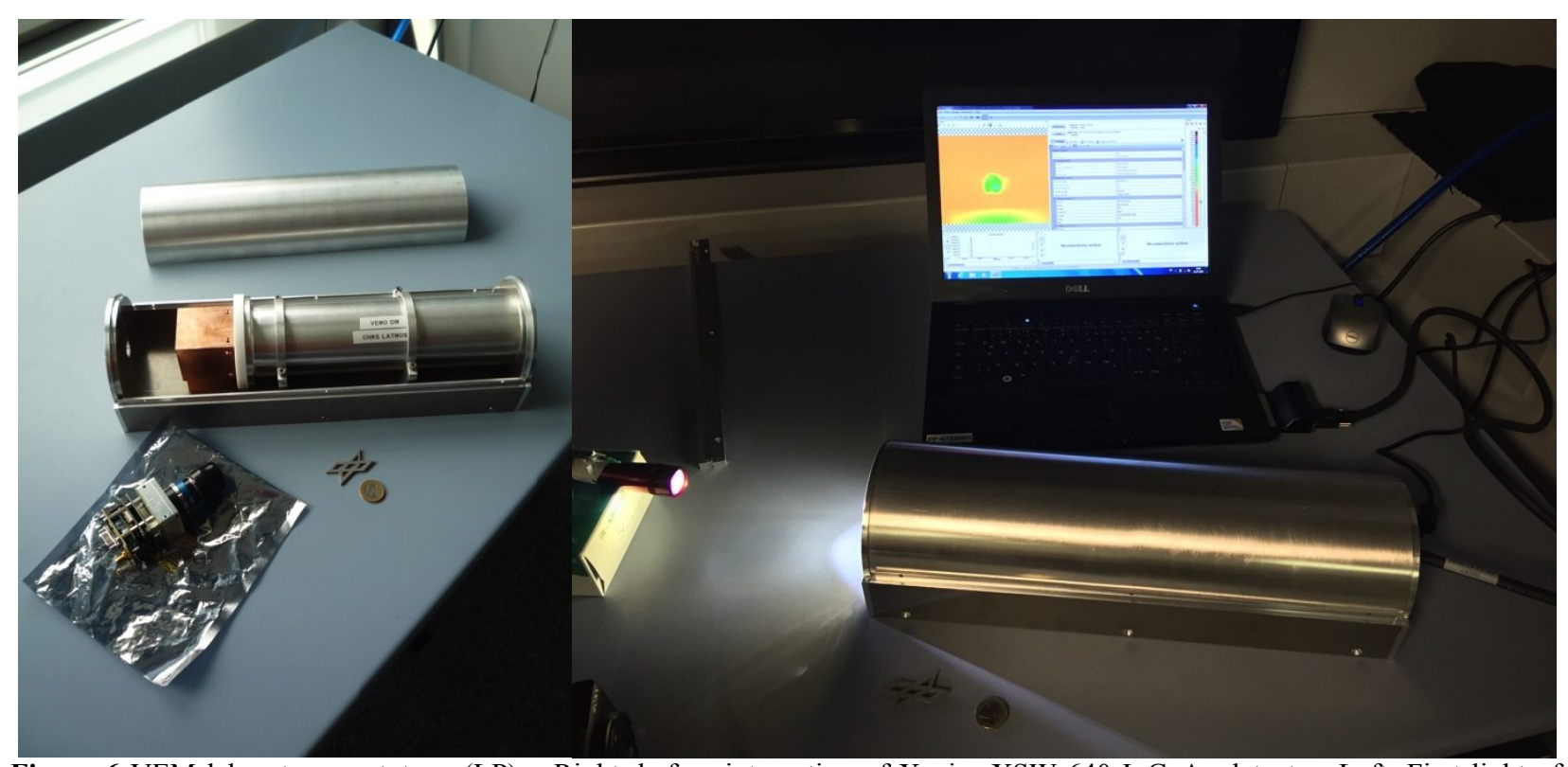

Figure 6 VEM laboratory prototype (LP) - Right: before integration of Xenics XSW-640 InGaAs detector. Left: First light of the VEM LP 


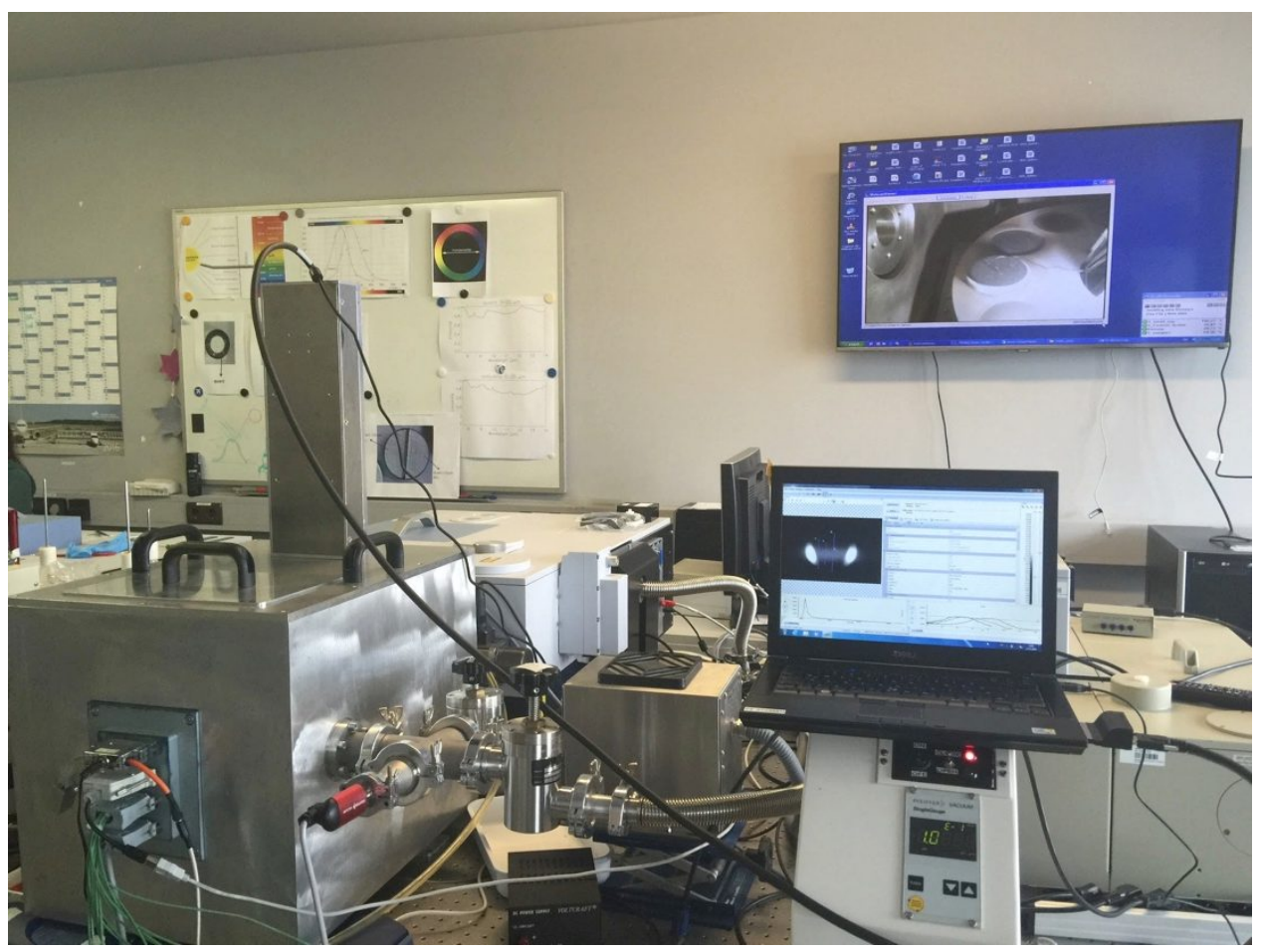

Figure 7 Preliminary performance testing with the VEM LP on the planetary simulation chamber at the PSL at DLR in Berlin. The screen on the wall shows the Venus analog target in the chamber

\section{SUPPORTING HIGH TEMPERATURE LABORATORY SPECTROSCOPY}

Building In the last decade the Planetary Emissivity Laboratory (PEL) of DLR in Berlin has provided spectral measurements of planetary analogues from the visible to the far-infrared range for comparison with remote sensing spacecraft/telescopic measurements of planetary surfaces [1-5]. Bi-directional reflection, transmission and emission spectroscopy are the techniques we used to acquire spectral data of target materials.

In fall 2015 we started upgrading our laboratory set-up, adding a new spectrometer, three external sources, and new detectors and beamsplitters to further extend the spectral range of measurements that can be performed in the laboratory. The new facility received the name of Planetary Spectroscopy Laboratory (PSL). Table 1 show the collection of detectors and beamsplitters available at PSL for spectroscopic measurements, to cover the entire spectral range from the UV to the FIR.

This upgrading was possible thanks to a $200 \mathrm{k} €$ contribution from the European Union's Horizon 2020 research and innovation programme under the grant agreement No 654208.

Our laboratory is one of the laboratory facilities offered under the TA (Transnational Access) programme of the Europlanet 2020 Research Infrastructure (RI), a $€ 9.95$ million project to integrate and support planetary science activities across Europe. The project is funded under the European Commission's Horizon 2020 programme; it was launched on 1st September 2015 and will run until 31 August 2019. More information under the website: http://www.europlanet2020-ri.eu/europlanet-2020-ri/europlanet-ta-facilities/ta2-distributed-planetary-simulation-facility-dpsf, http://www.europlanet-2020-ri.eu/TAs. 


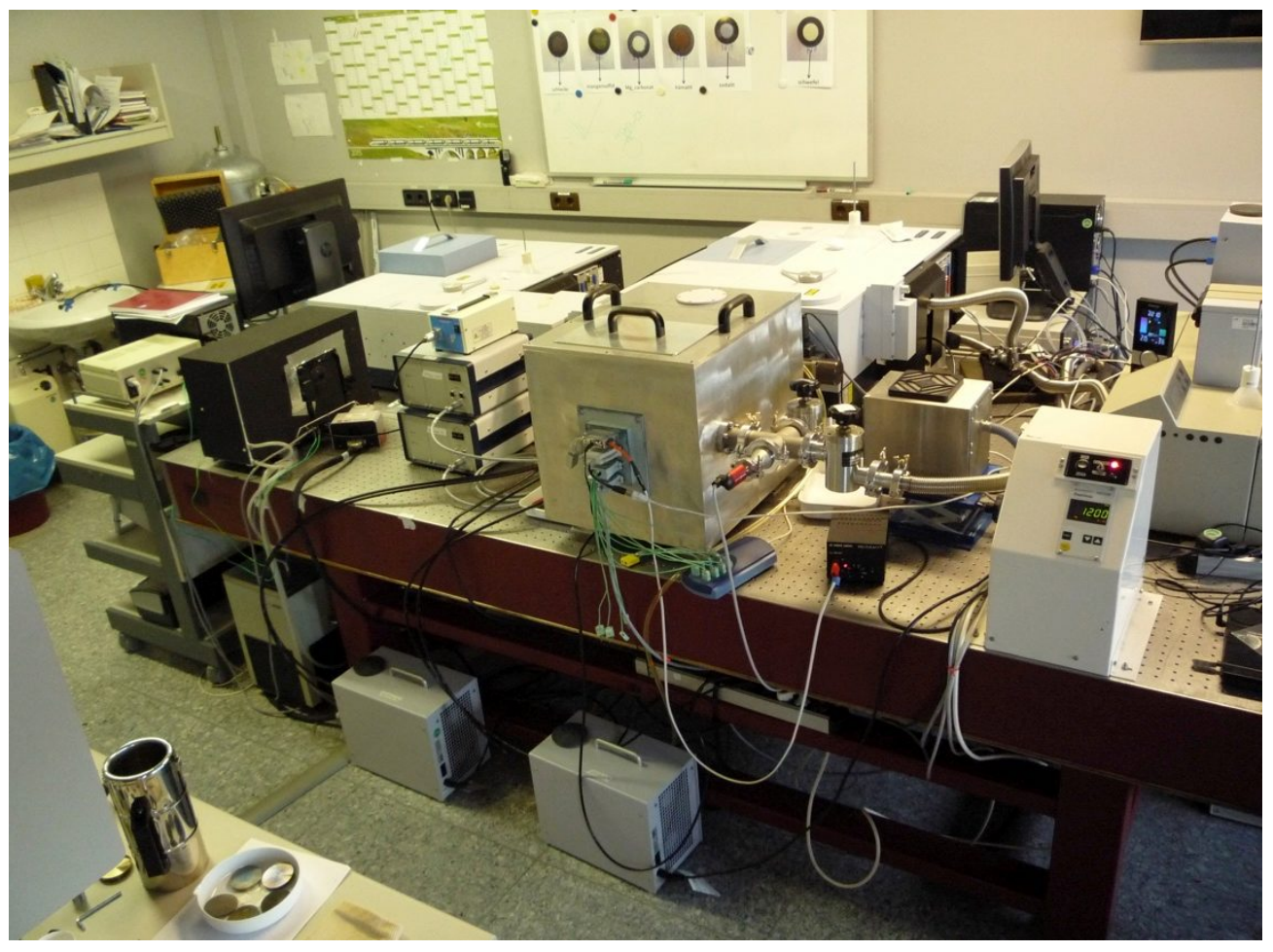

Figure 8 Current configuration at the Planetary Emissivity Laboratory

Building on the experienced collected in the last 3 years of laboratory experiments and considering the expected emissivity variation between felsic and mafic minerals with Venera and VEGA geochemical data, we have focused our analyses of a set of Venus analogue samples. Observations have suggested compositional variations correlated with geological features.

Studying surface composition based on only a small number of spectral channels in a narrow spectral range is very challenging. The task is further complicated by the fact that Venus has an average surface temperature of $460^{\circ} \mathrm{C}$. Spectral signatures of minerals are affected by temperature, so comparisons with mineral spectra obtained at room temperature can be misleading.

\subsection{Laboratory setup}

Figure 8 shows the optical table where the two FTIR instruments are operating at PSL, in an airconditioned room. The spectrometers are two Bruker Vertex $80 \mathrm{~V}$ that can be evacuated to $\sim 1 \mathrm{mbar}$. One spectrometer is equipped with aluminum mirrors optimized for the UV, visible and near-IR, the second features gold-coated mirrors for the near to far IR spectral range. Because the two instruments are identical apart of the mirrors, they can share the collection of detectors and beamsplitters we have in our equipment to cover a very wide spectral range. The instruments and the accessory units used are fully automatized and the data calibration and reduction are made with homemade DLR developed software. Table 1 list the spectral coverage of detectors and beamsplitters we have available at PSL.

To allow high precision transmission and reflectance measurements, three external sources have been added to the PSL set-up. A deuterium lamp is used to cover the UV $(0.2$ to $0.5 \mu \mathrm{m})$ spectral range. A $24 \mathrm{~V}$, water cooled, Tungsten lamp has been added for measurements in the VIS (0.4 to $1.1 \mu \mathrm{m})$ spectral range. Another high power Globar lamp ( $24 \mathrm{~V}$, water cooled) is used in the VNIR+TIR (1 to $16 \mu \mathrm{m})$ spectral range. A number of sample preparation and analysis tools and experiment sub-systems are available to the facility: a collection of hundreds of rocks and minerals, synthetic minerals, an Apollo 16 
lunar sample, several meteorites, set of sample holders for reflectance (plastic, aluminum or stainless steel), various sets of sieves, grinders, mortars, saw, scales, microscope, an oven $\left(20^{\circ}\right.$ to $\left.300^{\circ} \mathrm{C}\right)$, ultrapure water, wet chemistry materials, a second ovens $\left(30^{\circ}\right.$ to $\left.3000^{\circ} \mathrm{C}\right)$ for sample treatments, a press to produce pellets $(10 \mathrm{~mm}$ or $20 \mathrm{~mm}$ diameter), a large dry cabinet (moisture $<1 \%$ ) for sample storage, 3 small exsiccators (moisture $<20 \%$ ) for sample storage, a rotating device for producing intimate mixtures, purge gas generator for water and CO2 free air, liquid-nitrogen tank, an ultrasonic cleaning unit, 2 microscopes, air compressor pistol for cleaning. When enough sample material is available, the typical grain size separates that we produce for spectral measurements are $<25 \mu \mathrm{m}, 25-63 \mu \mathrm{m}, 63-125$ $\mu \mathrm{m}, 125-250 \mu \mathrm{m}$. Larger separates as well as slabs are also routinely measured

Table 1 List of detectors (left) and beamspitters (right) available at PSL

\begin{tabular}{|c|c|c|c|c|}
\hline Detector & $\begin{array}{l}\text { Spectral Range } \\
(\mu \mathrm{m})\end{array}$ & Operating T & Beamsplitter & $\begin{array}{l}\text { Spectral Range } \\
(\mu \mathrm{m})\end{array}$ \\
\hline GaP Diode & $0.2-0.55$ & Room T & $\begin{array}{l}\mathrm{UV} / \mathrm{VIS} / \mathrm{NIR} \\
\mathrm{CaF}_{2}\end{array}$ & $0.18-2.5$ \\
\hline Silicon Diode & $0.4-1.1$ & Room T & $\mathrm{Si}$ on $\mathrm{CaF}_{2}$ & $0.66-8.3$ \\
\hline $\begin{array}{l}\text { InGaAs } \\
\text { Diode }\end{array}$ & $0.7-2.5$ & Room T & $\begin{array}{l}\text { Ge on } \mathrm{KBr} \\
\text { (Wide) }\end{array}$ & $1-25$ \\
\hline $\mathrm{InSb}$ & $0.78-5.4$ & Liquid N2 & $\begin{array}{l}\text { Ge on } \mathrm{KBr} \\
\text { substrate }\end{array}$ & $1.2-25$ \\
\hline $2 \mathrm{x} \mathrm{MCT}$ & $0.8-16$ & Liquid N2 & Multilayer & $14.7-333$ \\
\hline $\begin{array}{l}\mathrm{MCT} / \mathrm{InSb} \\
\mathrm{SW}\end{array}$ & $1-16$ & Liquid N2 & $50 \mu \mathrm{m}$ Mylar & $181-666$ \\
\hline $\begin{array}{l}2 \mathrm{x} \\
\mathrm{DTGS} / \mathrm{KBr}\end{array}$ & $0.8-40$ & Room T & & \\
\hline DTGS/CsI & $0.8-55$ & Room T & & \\
\hline DTGS/PE & $14-1000$ & Room T & & \\
\hline
\end{tabular}

An external chamber (Figure 9) is attached to one of the FTIR spectrometers to measure the emissivity of solid samples. A shutter allows separating the spectrometer from the external chamber, which can be evacuated to the same pressure as of the spectrometer. If needed, an optical window (vacuum tight) can be mounted at the entrance of the emissivity chamber to operate while keeping the external chamber at ambient pressure under purged air or under inert gases. Sample targets are brought to measuring temperature using an induction heating system. Also, our sample cups and heating surfaces are made of stainless steel and heat the samples from below. Our high efficiency induction system heats the samples to temperatures from $320 \mathrm{~K}$ up to $900 \mathrm{~K}$. A sample carousel driven by a very precise stepper motor (computer controlled) allows measuring several consecutive samples without breaking the vacuum. A large number of temperature sensors in the emissivity chamber serve to monitoring sample as well as equipment and chamber temperature. A webcam is mounted in the emissivity chamber to monitor the heated sample and its vicinity.

With the Bruker A513 accessory on Vertex 80V, we can obtain bi-directional reflectance of minerals, with variable incidence and emission angles between $13^{\circ}$ and $85^{\circ}$. The viewing cone of the A513 has an 
aperture of $17^{\circ}$, small enough to define our measurements as bi-directional. We measure at room temperature, under purge or vacuum conditions, covering the 0.2 to above $200 \mu \mathrm{m}$ spectral range.

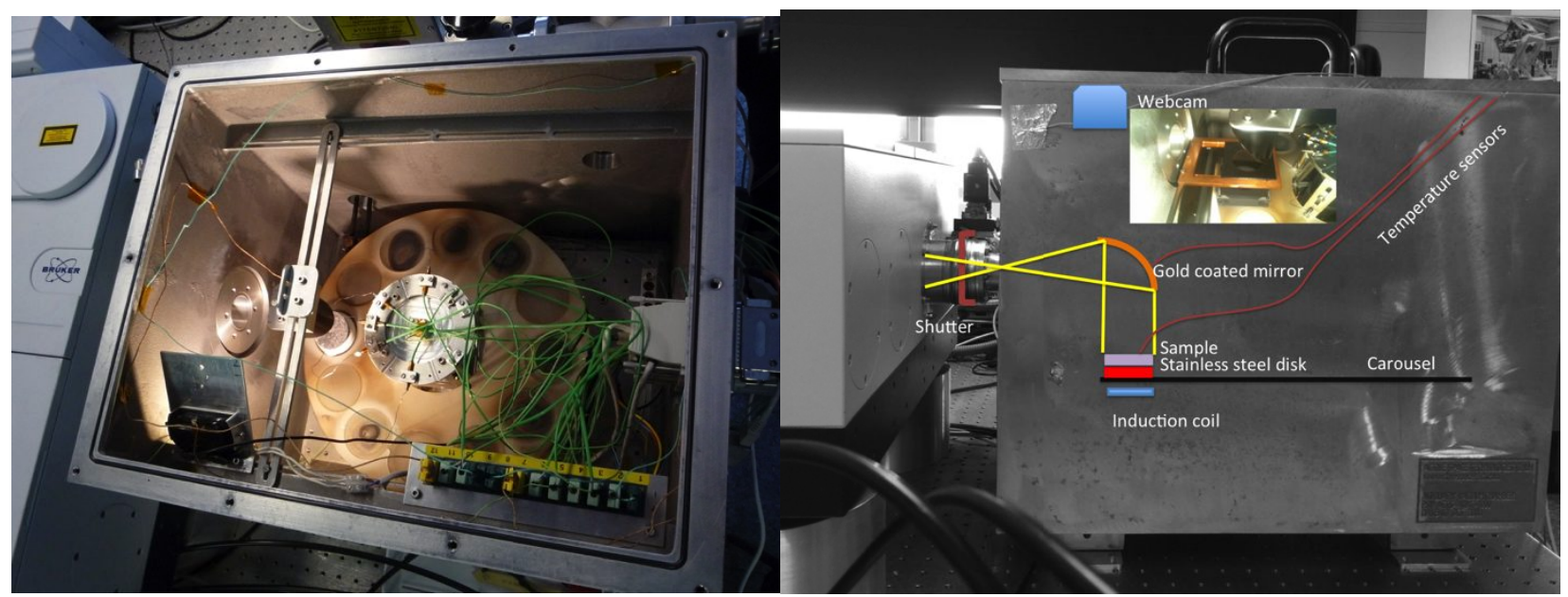

Figure 9 Left: External emissivity chamber at the Planetary Spectroscopy Laboratory (PSL), Right: Schematic view of the configuration and the major elements of the external emissivity chamber

The Bruker A480 parallel beam accessory mounted on the Vertex $80 \mathrm{~V}$ allows us to measure transmission of thin slabs, optical filters, optical windows, slabs, etc, in the complete spectral range from UV to FIR avoiding refraction, typical in this kind of measurements.

\subsection{Venus emissivity measurements}

The main feature of the PEL is a high-temperature chamber attached to the Vertex 80V that allows heating of samples to temperatures up to $1000 \mathrm{~K}$ under vacuum conditions (medium vacuum $\approx 10$ $100 \mathrm{~Pa}$ ) [8]. Samples are placed in steel cups equipped with type $\mathrm{K}$ thermopiles as temperature sensors. A copper induction coil installed in the chamber and connected to a Linntherm $1.5 \mathrm{~kW}$ induction system allows contactless heating of ferromagnetic sample cups by induction. Spectral coverage is achieved using a liquid nitrogen-cooled MCT detector and $\mathrm{KBr}$ beamsplitter for the spectral range up to $16 \mu \mathrm{m}$ and a DTGS detector with a multilayer beamsplitter for the remaining spectral range. Main components of the EU-financed upgrades are the InGaAs detector with matching beamsplitter, improved spectrometer electronics, and an optimization of the optical layout in the chamber. Upgrades began in October 2015 and will be completed by the end of 2016.

Building on the results from our laboratory experience and considering the expected emissivity variation between felsic and mafic minerals with Venera and VEGA geochemical data we have continued our analyses of Venus analogues materials, concentrating our efforts on slab samples for the moment.

Measuring emissivity at $1 \mu \mathrm{m}$ at Venus analogue temperatures is already very challenging for many reasons. For example, the emissivity of stainless steel increases strongly towards shorter wavelength at high temperatures. This results in a non-negligible contribution to total radiance from our sample cups. At the same time, many natural materials have a high transparency at $1 \mu \mathrm{m}$. To solve both issues at the same time, we are currently limiting ourselves to slabbed samples of materials with low transparency that are heated by placing them on a stainless steel disk completely obscured by the sample.

Extending the measurements below $1 \mu \mathrm{m}$ adds significant new challenges. The main challenge is the very low thermal emission due to the fact that we are on the steep flank of the Planck curve, where between $1.18 \mu \mathrm{m}$ and $0.8 \mu \mathrm{m}$ the signal drops by more than two orders of magnitude. 
Therefore even the use of a steel disk under the slab for heating seems not to be enough. The glow of the steel scattered in the chamber becomes a non-negligible noise term that results in an apparent increase of the emissivity around $0.9 \mu \mathrm{m}$.

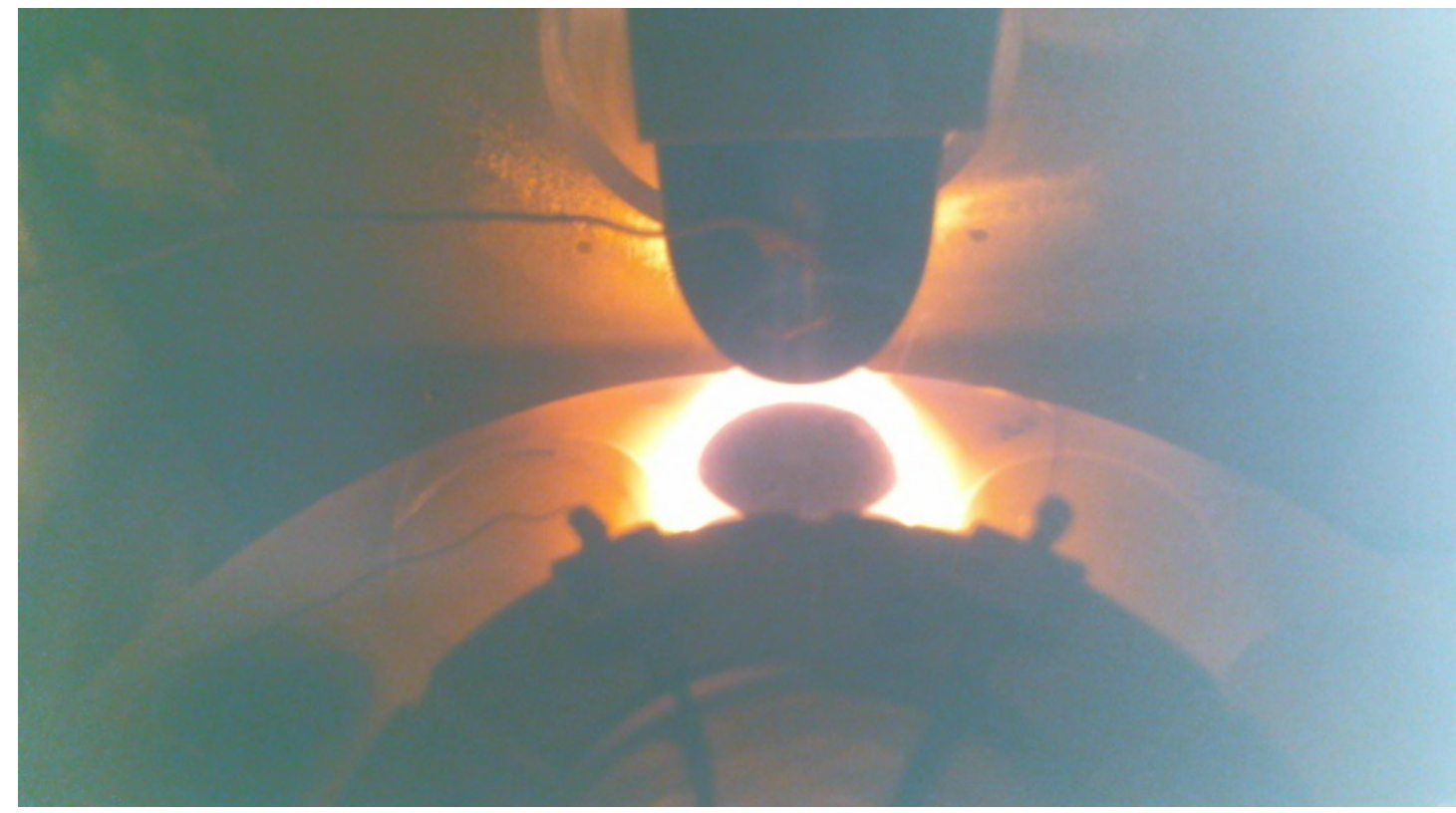

Figure 10 Steel disk glowing when heated above $700 \mathrm{~K}$

Our initials attempts to measure powdered samples in stainless steel cups (needed because we use the induction system as heater) were unsatisfying, mostly because steel, when heated to high temperatures needed to bring the granular sample surface to above $700 \mathrm{~K}$, is strongly glowing, also adding a large amount of radiation in the visible spectral range we are exploring, as can be seen from Figure 11 . We furtherly noticed that granular material heated at high temperature $(670 \mathrm{~K}$ to $750 \mathrm{~K})$ in vacuum tends to be very transparent, with the result that for most of the samples we were observing the emission from the sample cup (high T steel glowing) instead of the emitting sample powder. We therefore decided to concentrate our efforts to slab samples. However, the problem of the glowing steel heater remained, and after some test and discussions with our thermal engineers, we took the decision to build a ceramic enclosure, where to embed the steel disk (needed to heat the samples), so to reduce the disturbing effect coming from the glowing steel. Figure 12 shows the ceramic enclosure and the steel disk we use to embed inside. This ceramic enclosure is made of FRIALIT GP79 $\left(\mathrm{Si}_{3} \mathrm{~N}_{4}\right)$, whose emissivity in the VIS spectral range is much reduced. We heated the ceramic material to temperatures above $700 \mathrm{~K}$ to test its emissivity in the visible spectral range. The left panel of figure 13 shows a snapshot taken with a webcam internal to the emissivity chamber: the ceramic enclosure, heated at $750 \mathrm{~K}$, is slightly emitting in the VIS, much lesser than the steel disk used previously. We collected measurements of the VIS radiation coming from the ceramic enclosure, so that we could calibrate its emissivity. Figure 14 shows the calibrated emissivity for the ceramic enclosure, for measurements taken with surface temperature of $400^{\circ} \mathrm{C}(673 \mathrm{~K})$ and $460^{\circ} \mathrm{C}(733 \mathrm{~K})$. 


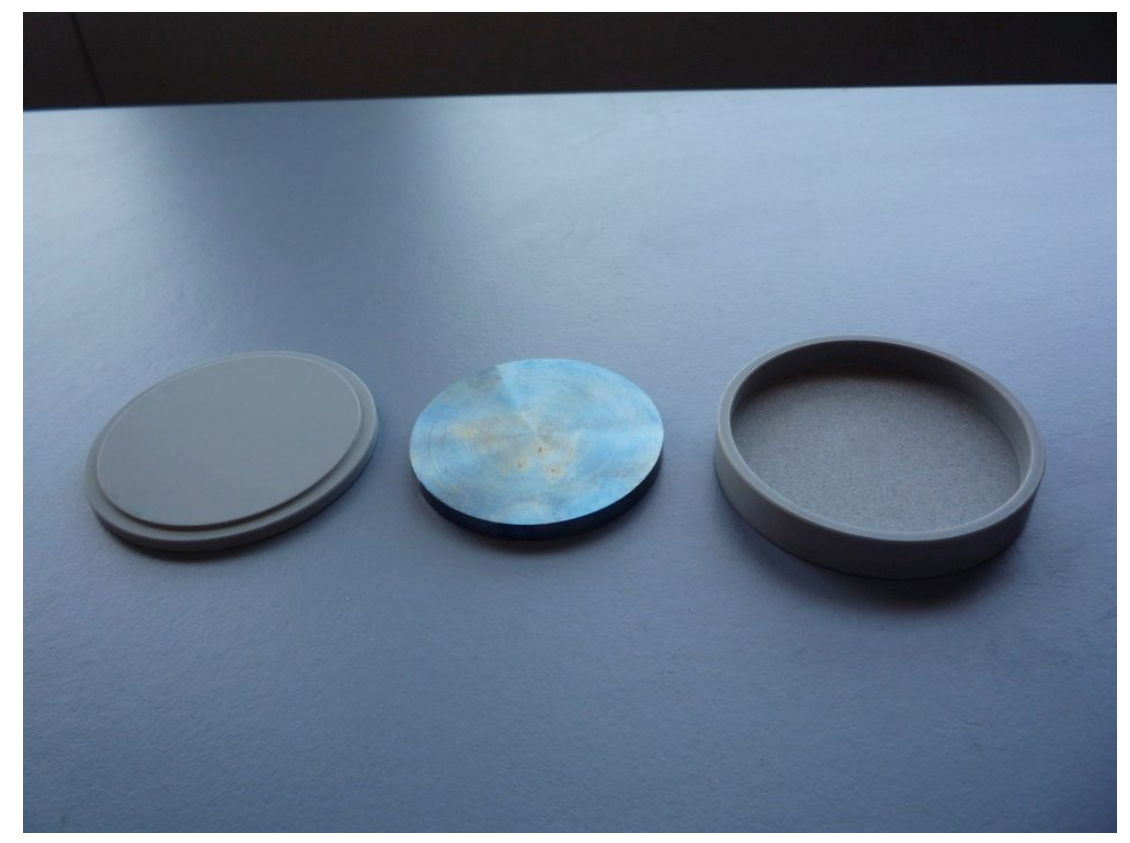

Figure 11 Steel disk and the ceramic enclosure we use to mask its glowing in the VIS

Once we verified that our heating system, made of a steel disk embedded in a ceramic enclosure was responding to our requirements of having less perturbation as possible coming from the heating itself on the emissivity measurements of Venus analogue slabs in the visible spectral range, we could move forward, heating a slab of hematite (one of our Venus analogue samples we always used as a reference for the testing of our set-up) with this custom made heating system. The right panel of figure 13 shows a picture taken with the internal webcam, for the hematite slab heated (on the surface) at $730 \mathrm{~K}$, where one can see how little is the visible irradiation coming from the heating block.

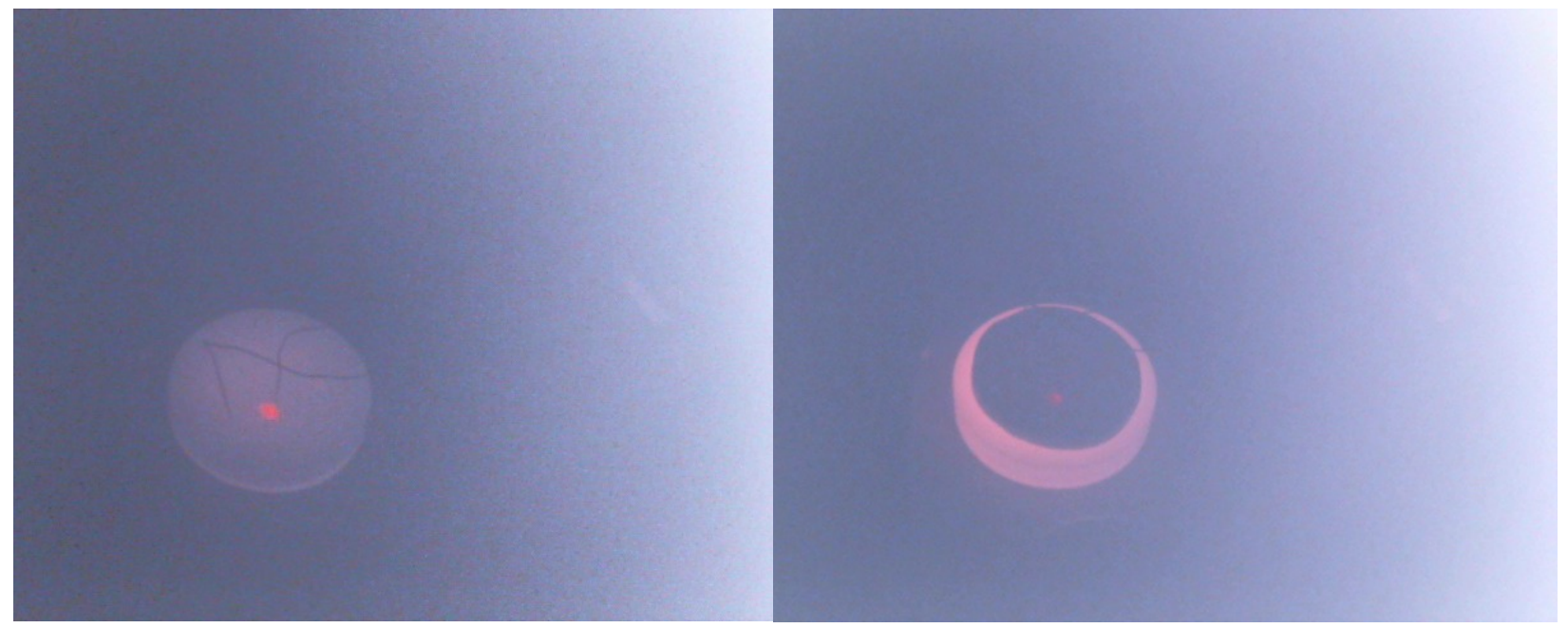

Figure 12 Webcam picture of the ceramic enclosure (left) and the hematite slab on the ceramic enclosure (right) heated at $750 \mathrm{~K}$

This system showed to perform following our requirements and it is stable and allows reproducibility of the results, hence we took the action of freezing it as our standard set-up for emissivity measurements of Venus analogue slabs in the visible spectral range. 


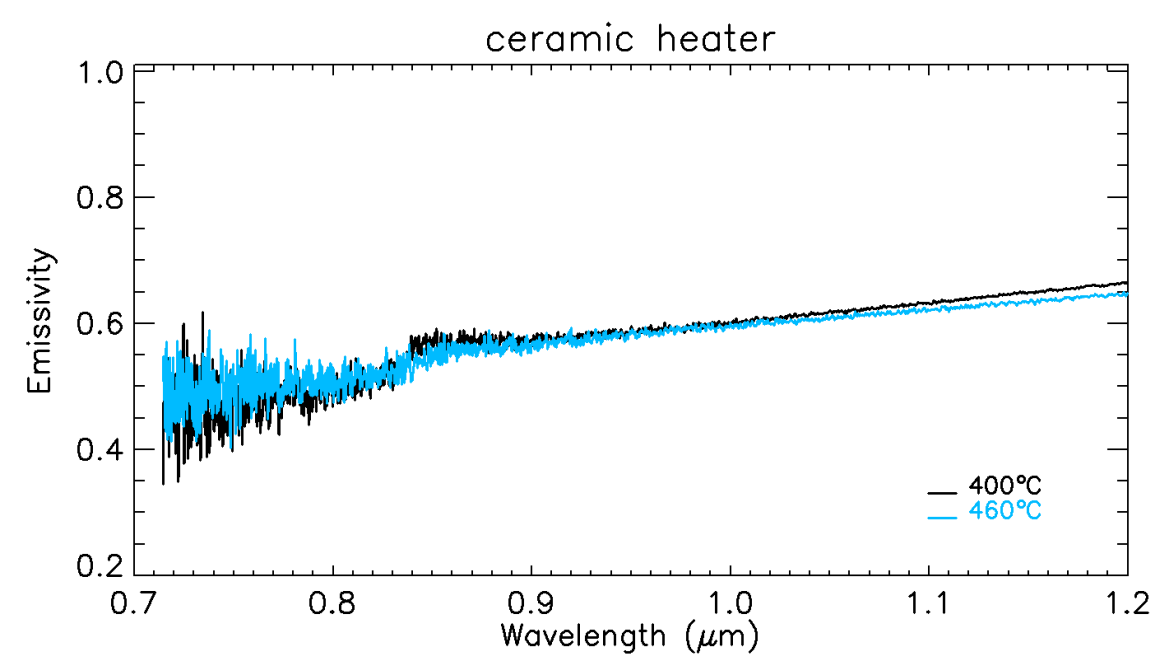

Figure 13 Calibrated emissivity spectra for the ceramic enclosure heated at $400^{\circ} \mathrm{C}$ and $460^{\circ} \mathrm{C}$

\section{OUTLOOK}

Observing the surface of Venus in the near infrared requires a dedicated instrument. VIRTIS observations have successfully demonstrated that important information can be extracted from the windows in the visible portion of the spectrum, but the design of the instrument limited its use for surface investigations. Deploying an instrument like VEM in orbit or on an aerial platform will provide new insights into the mineralogy of Venus. In combination with a high-resolution radar mapper that provides accurate topographic data as planned for the VERITAS mission or for the ESA EnVision mission proposal, this will allow a global mapping of the surface composition at a spatial scale of approximately $50 \mathrm{~km}$ Combining the near infrared data with radar derived geological information will allow further conclusions on the evolution of Venus to be drawn.

\section{REFERENCES}

[1] Smrekar S. et al., "VERITAS (Venus Emissivity, Radio Science, InSAR, Topography and Spectroscopy): A Proposed Discovery Mission “LPSC, \#1903 (2016)

[2] Ghail RC, Wilson C, Galand M, et al., "EnVision: taking the pulse of our twin planet.”,Exp Astron 33:337-363. doi: 10.1007/s10686-011-9244-3 (2012)

[3] Wilson C.F. et al., "Analysis of thermal emission from the nightside of Venus at 1.51 and $1.55 \mu \mathrm{m}$ " Icarus, 814817 (2009)

[4] Mueller, N., et al., "Venus surface thermal emission at $1 \mu \mathrm{m}$ in VIRTIS imaging observations: Evidence for variation of crust and mantle differentiation conditions." Journal of Geophysical Research: Planets 113.E5 (2008).

[5] Helbert J, Müller N, Kostama P, et al., „,Surface brightness variations seen by VIRTIS on Venus Express and implications for the evolution of the Lada Terra region, Venus." Geophys Res Lett 35:1-5. doi: 10.1029/2008GL033609 (2008)

[6] Kappel D, Haus R, Arnold G, "Error analysis for retrieval of Venus' IR surface emissivity from VIRTIS/VEX measurements."Planet Space Sci 113-114:49-65. doi: 10.1016/j.pss.2015.01.014 (2015)

[7] Kappel D, Arnold G, Haus R, "Multi-spectrum retrieval of Venus IR surface emissivity maps from VIRTIS/VEX nightside measurements at Themis Regio." Icarus 265:42-62. doi: 10.1016/j.icarus.2015.10.014 (2016) 
[8] Peter G, Helbert J, Hiesinger H, et al., "Developing of MERTIS as an advanced process from the study up to the flight model.” In: Strojnik Scholl M, Páez G (eds) SPIE Opt. ... p 886707 (2013)

[9] Wendler D., „Design, Fertigung und Test eines Entwicklunsmodells zur Untersuchung des optischen Designs des Venus Emissivity Mappers (VEM) unter Verwendung des Raspberry Pi“ ELIB DLR, 1. Juli 2016, http://elib.dlr.de/101033/ (2015)

[10] Maturilli, A. and Helbert, J., "Emissivity measurements of analogue materials for the interpretation of data from PFS on Mars Express and MERTIS on Bepi-Colombo" PSS, Vol. 54, pp. 1057-1064, (2006)

[11] Maturilli, A., Helbert, J., and Moroz L., "The Berlin Emissivity Database (BED)" PSS, Vol. 56, pp. 420-425, (2008)

[12] Helbert, J. and Maturilli, A., "The emissivity of a fine-grained labradorite sample at typical Mercury dayside temperatures" EPSL, Vol. 285, pp. 347-354, (2009)

[13] Maturilli A, Helbert J, "Characterization, testing, calibration, and validation of the Berlin emissivity database." Journal of Applied Remote Sensing, (2014)

[14] Maturilli A, Helbert J, St. John JM, Head III JW, Vaughan WM, D'Amore M, Gottschalk M, Ferrari S, "Komatiites as Mercury surface analogues: Spectral measurements at PEL." EPSL, Vol. 398, pp. 58-65, (2014). 